\title{
Single vs. combination immunotherapeutic strategies for glioma
}

\section{Mayuri Chandran, Marianela Candolfi, Diana Shah, Yohei Mineharu, Vivek Yadav, Carl Koschmann, Antonela S. Asad, Pedro R. Lowenstein \& Maria G. Castro}

To cite this article: Mayuri Chandran, Marianela Candolfi, Diana Shah, Yohei Mineharu, Vivek Yadav, Carl Koschmann, Antonela S. Asad, Pedro R. Lowenstein \& Maria G. Castro (2017): Single vs. combination immunotherapeutic strategies for glioma, Expert Opinion on Biological Therapy, DOI: $10.1080 / 14712598.2017 .1305353$

To link to this article: http://dx.doi.org/10.1080/14712598.2017.1305353

Accepted author version posted online: 13

Mar 2017.

Submit your article to this journal $₫$

Q View related articles $₫$

View Crossmark data \lceil 
Publisher: Taylor \& Francis

Journal: Expert Opinion on Biological Therapy

DOI: $10.1080 / 14712598.2017 .1305353$

\section{Review}

\section{Single vs. combination immunotherapeutic strategies for glioma}

Mayuri Chandran ${ }^{1,2}$, Marianela Candolfi ${ }^{3}$, Diana Shah ${ }^{1,2}$, Yohei Mineharu ${ }^{4}$, Vivek Yadav ${ }^{1,2}$, Carl Koschmann $^{1,2}$, Antonela S. Asad ${ }^{3}$, Pedro R. Lowenstein ${ }^{1,2}$ and Maria G. Castro ${ }^{1,2}$

${ }^{1}$ Department of Neurosurgery, The University of Michigan School of Medicine, MSRB II, RM 4570C, 1150 West Medical Center Drive, Ann Arbor, MI 48109-5689, USA.

${ }^{2}$ Department of Cell and Developmental Biology, The University of Michigan School of Medicine, Ann Arbor, MI, USA.

${ }^{3}$ Instituto de Investigaciones Biomédicas (CONICET-UBA), Facultad de Medicina, Universidad de Buenos Aires, Buenos Aires, Argentina.

${ }^{4}$ Department of Neurosurgery, Kyoto University Graduate School of Medicine 54 Kawahara-cho, Shogoin, Sakyo-ku, Kyoto 606-8507, Japan.

Key words: Glioma, immunotherapy, gene therapy, cancer vaccines, immune checkpoint blockade.

Correspondence should be addressed to: Maria G Castro (mariacas@umich.edu). 
Tel: 734.764 .0850

\section{Article Highlights:}

- Immunotherapy for malignant glioma is complicated by several factors that may limit the therapeutic efficacy of monotherapies: (i) gliomas are characterized by a high degree of inter-tumor and intra-tumor cellular and molecular heterogeneity; (ii) gliomas have been shown to induce immunosuppression through secreted factors, expression of immunosuppressive checkpoints, and infiltration of immunosuppressive cells.

- Use of single arm immunotherapies may induce selection and continued growth of poorly immunogenic tumor cells.

- There are currently several immunotherapies that show promising results in preclinical and clinical studies, including immunosuppressive checkpoint inhibition, immunestimulatory gene therapy, antitumor vaccination, oncolytic virotherary, passive antibody therapy, and adoptive $\mathrm{T}$ cell therapy.

- Combinations of currently available immunotherapies have significant potential to improve efficacy of treatment.

Combinations of checkpoint inhibitors are currently being tested in GBM patients (NCT02017717, NCT02311920, NCT02794883). 
- Preclinical studies utilizing two-arm combination gene therapy, comprising the conditional cytotoxic gene TK and the cytokine Flt3L resulted in tumor-specific immune responses, long term survival and immunological memory in glioma bearing rats and mice. This combined immune-gene therapy approach is currently being tested in human GBM clinical trials (NCT01811992).

- Combinations involving more than one type of immunotherapeutic approach are also promising strategies. For example, combining DC vaccination with TK/Flt3L gene therapy resulted in $90 \%$ long-term survival of rats with large syngeneic brain tumors, a significant increase in survival when compared to either therapy alone.

- Combining therapeutic approaches which induce immunogenic tumor cell death with immunotherapies has also shown promise in preclinical and clinical settings. An ongoing Phase I clinical trial is testing the efficacy of oncolytic virotherapy with an oncolytic herpes simplex virus (oHSV) engineered to express the cytokine IL-12 in patients with recurrent/progressive GBM, anaplastic astrocytoma, or gliosarcoma (NCT02062827). 


\begin{abstract}
Introduction: Malignant gliomas are highly invasive tumors, associated with a dismal survival rate despite standard of care, which includes surgical resection, radiotherapy and chemotherapy with temozolomide (TMZ). Precision immunotherapies or combinations of immunotherapies that target unique tumor-specific features may substantially improve upon existing treatments.
\end{abstract}

Areas covered: Clinical trials of single immunotherapies have shown therapeutic potential in high-grade glioma patients, and emerging preclinical studies indicate that combinations of immunotherapies may be more effective than monotherapies. In this review, the authors discuss emerging combinations of immunotherapies and compare efficacy of single vs. combined therapies tested in preclinical brain tumor models.

Expert opinion: Malignant gliomas are characterized by a number of factors which may limit the success of single immunotherapies including inter-tumor and intra-tumor heterogeneity, intrinsic resistance to traditional therapies, immunosuppression, and immune selection for tumor cells with low antigenicity. Combination of therapies which target multiple aspects of tumor physiology are likely to be more effective than single therapies. While a limited number of combination immunotherapies are described which are currently being tested in preclinical and 
clinical studies, the field is expanding at an astounding rate, and endless combinations remain open for exploration.

\section{Introduction}

Gliomas are highly invasive tumors which often occur in the cerebral hemispheres ${ }^{1}$. Glioblastoma Multiforme (GBM), the most common and aggressive form of glioma, has a 5year survival rate of about $5 \%{ }^{2}$, creating a need for aggressive and effective therapies. Current standard of care includes surgical resection followed by radiotherapy and the alkylating agent temozolomide $(\mathrm{TMZ})^{3}$. However, surgical resection is limited by the diffuse, infiltrative nature of these tumors, and median surviyal following surgery, radiotherapy, and TMZ remains dismal ${ }^{3}$. The development of immunotherapies which target unique features of glioma may significantly improve therapeutic outcomes. It has been extensively demonstrated that it is not possible to mount an effective adaptive immune response against antigens located within the brain parenchyma $^{4-7}$. However, research from our group and others has established that the central nervous system is not an immune-privileged site as previously thought ${ }^{8-11}$. Antigen presenting cells (APCs) are indeed present in the normal brain ${ }^{12}$, albeit at very low levels. Immune cells can traffic from the CNS to the draining cervical lymph nodes ${ }^{13}$, and the BBB is permeable to immune cells under conditions of inflammation ${ }^{14}$, opening the door to effective immunotherapies for tumors located within the brain. One main hurdle that needs to be overcome is the highly 
immunosuppressive nature of the tumor microenvironment ${ }^{14,15}$, which hampers the efficacy of immunotherapies.

\subsection{Molecular heterogeneity in glioma}

Recent advances in molecular characterization of gliomas have allowed for higher resolution classification, facilitating the development of precision immunotherapies or combinations of immunotherapies for GBM. Traditional histopathological methods of diagnosis rely on morphological classification of gliomas as astrocytomas, oligodendrogliomas, or oligoastrocytomas, a process which is complicated by morphological ambiguity and interobserver variability ${ }^{16}$. The new 2016 WHO classification of CNS tumors incorporates both histopathological features and molecular markers into its updated classification scheme, increasing objectivity of classification and homogeneity of diagnostic groupings ${ }^{17}$. The 2016 WHO classification distinguishes primary and secondary GBM based on IDH mutation status. While IDH wild type and IDH mutant tumors appear histologically similar, they arise from distinct cells of origin and likely represent separate disease entities ${ }^{18}$. Primary GBMs, which arise de novo mostly in elderly patients, are defined by wild type IDH1 status, while secondary GBMs, which usually occur in younger patients and are associated with a more favorable prognosis, display mutant IDH $1^{19}$. Pediatric gliomas are characterized by the presence of PDGFR amplification ${ }^{20}$ and H3F3A mutation ${ }^{21,22}$. Other molecular markers with prognostic value are methylation of the MGMT gene and codeletion of chromosome arms $1 \mathrm{p}$ and $19 \mathrm{q}$, which are both independently associated with favorable clinical prognosis ${ }^{23}$, and ATRX mutations, which result in greater responsiveness to double stranded DNA-damaging agents in preclinical studies ${ }^{24}$. 
Large scale clustering of recurrent genomic alterations in GBM into proneural, neural, classical, and mesenchymal subtypes reveals differences in treatment efficacy of chemotherapy and radiotherapy ${ }^{25}$. However, clinically relevant molecular characterization of tumors is complicated by the heterogeneity of molecular markers within a single tumor. Recent studies reveal that individual GBMs contain a mixture of cells corresponding to different subtypes; genetic signatures from cells in one tumor could be classified into more than one subtype in 5 out of 5 tumors sampled in one study ${ }^{26}$ and 6 out of 10 tumors sampled in another ${ }^{27}$. The striking diversity of tumor cells across different gliomas and within a particular tumor suggests that single therapies are less likely to be effective. Furthermore, treatment using a therapy which targets a single tumor specific antigen might eliminate highly antigenic tumor cells, applying a selective pressure in favor of variants which escape the immune system through poor antigenicity $^{28,29}$. A trial of the EGFRvIII peptide vaccine in patients with EGFRvIII-expressing GBM revealed that $82 \%$ of recurrent tumors had lost EGFRvIII expression ${ }^{29}$, suggesting that tumors may lose their antigenic epitopes over time.

\subsection{Immunosuppression in glioma}

The success of immunotherapies may be limited by a number of immunosuppressive mechanisms employed by GBM; understanding and targeting these mechanisms will likely broaden the therapeutic potential of immunotherapies. Glioma cells overexpress secreted immunosuppressive factors including TGF- $\beta$, IL-10, and VEGF (which limits antitumor immunity through defective dendritic cell (DC) maturation in addition to its role in neo-

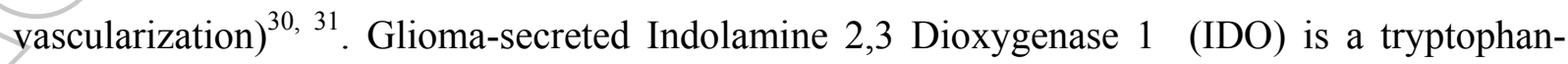
metabolizing enzyme that inhibits effector $\mathrm{T}$ cell function and increases the recruitment of immunosuppressive regulatory $\mathrm{T}$ cells $\left(\mathrm{T}_{\text {regs }}\right)^{32}$. It has been found that $\mathrm{T}_{\text {regs, }}$ which 
physiologically inhibit $\mathrm{T}$ cell activation to prevent autoimmunity, represent an increased fraction of the $\mathrm{CD} 4+\mathrm{T}$ cell population in patients with malignant glioma, resulting in immunosuppression $^{33}$. In addition to $T_{\text {regs, }}$ gliomas have increased infiltration of other immunosuppressive cells such as tumor associated macrophages (TAMs), which enhance tumor invasiveness and secrete immunosuppressive cytokines ${ }^{34}$, and myeloid-derived suppressor cells (MDSCs), which suppress $\mathrm{T}$ cell functions and promote the expansion of $\mathrm{T}_{\text {regs }} 35,36$

Cell surface molecules can further dampen antitumor immunity; GBM cells which express FasL induce apoptosis in $\mathrm{T}$ lymphocytes which express Fas ${ }^{37}$. Gliomas also strongly express B7$\mathrm{H} 1$, or $\mathrm{PDL}^{38}$, inhibiting CD4+ and $\mathrm{CD} 8+\mathrm{T}$ cell activation and mediating $\mathrm{T}$ cell exhaustion through the activation of Programmed Cell Death Protein $1(\mathrm{PD}-1)^{39}$. This process of $\mathrm{T}$ cell exhaustion results in decreased cytokine expression and effector function and upregulation of multiple immunosuppressive markers such as immunological checkpoints PD-1, Tim-3, and LAG- ${ }^{40}$. Cytotoxic T lymphocyte antigen 4 (CTLA4), a marker expressed on activated T cells and $\mathrm{T}_{\text {regs }} 41,42$ binds with high affinity to CD80 and CD86 receptors, blocking costimulatory signaling for $\mathrm{T}$ cell activation ${ }^{43-45}$. Because these markers limit the ability of the immune system to mount a successful antitumor response, and therapeutic strategies using blocking antibodies that target these proteins have proven efficacious for other tumors, such as melanoma, immunosuppressive checkpoints are attractive targets for GBM immunotherapy.

\section{Single immunotherapies for glioma}

Several approaches to immunotherapy for glioma are currently being tested, including immunosuppressive checkpoint blockade, immunostimulatory gene therapy, active immunotherapy through vaccination, passive antibody therapy, and adoptive immunotherapy 
through $\mathrm{T}$ cell transfer or CAR $\mathrm{T}$ cells ${ }^{46-49}$. A recent review by our group provides a comprehensive summary of currently tested single immunotherapies and their efficacies in preclinical and clinical studies ${ }^{50}$. Several immunotherapies show impressive antitumor activity in preclinical studies but demonstrate limited efficacy in clinical trials. Below we highlight two examples of single immunotherapies which demonstrate promising results in early studies but mixed results in large scale clinical trials.

\subsection{EGFRvIII peptide vaccine}

Peptide vaccination strategies aim to generate a tumor-specific adaptive immune response by targeting epitopes of tumor associated antigens, which are found in normal tissues but overexpressed in tumors, or tumor-specific antigens, which occur only in tumors. Commonly occurring mutations in glioma are excellent targets for peptide vaccination. One such target is epidermal growth factor receptor variant III (EGFRvIII); the EGFRvIII mutation is oncogenic in GBM, and results from an in-frame deletion of the extracellular domain of the protein, which creates a neoepitope $^{51}$. A phase II multicenter trial of the EGFRvIII peptide vaccine in patients with EGFRvIII-expressing GBM resulted in median overall survival (OS) of 26.0 months, greater than that of the control group ${ }^{29}$. However, at tumor recurrence $82 \%$ of patients did not exhibit EGFRvIII expression, suggesting that immune selection for tumor cells which lack expression of the targeted antigen may limit the success of single peptide vaccines ${ }^{29}$. Another recent phase II multicenter trial of the EGFRvIII peptide vaccine resulted in median OS of 21.8 months and median progression free survival (PFS) of 9.2 months, greater than OS and PFS reported in patients receiving standard therapy with surgery, chemoradiation, and $\mathrm{TMZ}^{52}$. However, it is likely that the phase II study, which compared EGFRvIII peptide vaccination to historical controls, overestimated therapeutic effect; the phase III study of the EGFRvIII peptide 
vaccine with GM-CSF was discontinued based on the determination by the Data Safety and Monitoring Board that continuation of the study would not reach statistical significance for overall survival in patients with minimal residual disease ${ }^{53}$.

2.2 Dendritic cell vaccines pulsed with peptides

DC vaccines take advantage of the fact that DCs are highly efficient APCs that can activate CD4, CD8, NK and NKT cells, inducing strong anti-tumor immunity ${ }^{46,54}$. DC vaccines are prepared from autologous mononuclear cells that are pulsed with peptides from tumor-associated antigens or whole tumor lysates. Interestingly, it has been shown that efficacy of DC immunotherapy correlates with glioma gene expression profile. In a clinical study of tumorlysate pulsed DCs, patients with the mesenchymal gene expression profile, which is associated with increased inflammatory infiltrates ${ }^{25}$, demonstrated increased survival when compared to historical controls of the mesenchymal subtype; patients with the proneural gene expression profile demonstrated no difference in overall survival when compared to historical controls of the proneural subtype ${ }^{55}$. A phase I/I trial evaluated the efficacy of a DC vaccine pulsed with synthetic peptides from glioma associated antigens (EphA2, IL13R $\alpha 2$, YKL-40, and gp100) in patients with recurrent malignant glioma ${ }^{56} .41 \%$ of patients demonstrated progression free survival for at least 12 months, and $9 \%$ of patients experienced objective clinical tumor regression 56 . The ICT-107 DC vaccine is pulsed with six synthetic peptides (HER2, TRP-2, gp100, MAGE-1, IL13R $\alpha 2$ and AIM-2) expressed on gliomas and overexpressed in glioma stem cells. In a phase II study of this vaccine, 17 patients with newly diagnosed GBM were treated with ICT-107, resulting in a median PFS of 16.9 months and median OS of 38.4 months $^{57}$. A phase III trial to test the efficacy of ICT-107 in HLA-A2-positive patients with newly diagnosed GBM (NCT02546102) is ongoing. Another phase III trial of an autologous DC vaccine, referred 
to as DCVax-Brain, was conducted for newly diagnosed patients with GBM (NCT00045968): patient-derived DCs were pulsed with the same set of six synthetic peptides included in the ICT107 vaccine (HER2, TRP-2, gp100, MAGE-1, IL13R $\alpha 2$ and AIM-2) ${ }^{58}$. Treatment efficacy was compared with placebo control, demonstrating limited clinical benefit.

\section{Combination immunotherapeutic strategies for glioma}

Combinations of immunotherapies may improve upon single immunotherapies through synergistic effects, ability to address heterogeneity of tumors, and limiting immune selection for cells which evade the immune system. While the following section describes a limited number of currently tested combinations of immunotherapies, the field is brimming with possibilities. Many other combinations are currently in the pipeline, and countless new strategies are open for exploration.

3.1 Combination strategies utilizing immunosuppressive checkpoint inhibitors

\subsubsection{Combinations of multiple checkpoint inhibitors}

Gliomas co-opt physiological strategies for preventing self-reactivity, dampening the antitumor immune response. Blocking these immunosuppressive checkpoints may allow for enhanced antitumor reactivity. Checkpoint inhibitors which target CTLA4 (an early checkpoint in the immune response which blocks costimulatory signaling) or the PD1/PDL1 axis (which mediates $\mathrm{T}$ cell exhaustion) are good candidates for combination immunotherapy. Preclinical studies by Wainwright et al provide promising evidence supporting therapeutic application of multiple checkpoint blockade. IDO inhibition with 1-methyltryptophan (1-MT) administered with anti-CTLA4 and anti-PDL1 monoclonal antibodies resulted in 100\% durable survival of mice implanted with GL261 glioma cells in comparison to $20 \%$ durable survival in mice treated 
with 1-MT alone ${ }^{59}$. Furthermore, triple therapy decreased the number of tumor infiltrating $\mathrm{T}_{\text {regs }}$ to a greater extent than treatment with 1-MT alone or combination of 1-MT with either CTLA4 blockade or PDL1 blockade ${ }^{59}$. Clinical studies of an anti-CTLA4 antibody (ipilimumab) combined with an anti-PD1 antibody (nivolumab) in patients with melanoma resulted in a $40 \%$ objective response rate, exceeding response rates in treatment with either ipilimumab or nivolumab alone ${ }^{60}$. Current clinical trials are testing the safety and efficacy of nivolumab combined with ipilimumab in GBM (NCT02017717, NCT02311920). Other clinical trials are testing different antibodies which target CTLA4 (tremelimumab) and PDL1) (durvalumab) in recurrent malignant glioma (NCT02794883), and nivolumab in combination with the anti-LAG3 antibody urelumab (NCT02658981) for GBM. One potential drawback of checkpoint inhibitors is the occurrence of treatment-related adverse events; the study of ipilimumab and nivolumab in patients with melanoma revealed treatment-related adverse events including rash, pruritus, fatigue, and diarrhea in $93 \%$ of patients who received concurrent treatment, and serious adverse events including hepatic, gastrointestinal, and renal events in $49 \%$ of patients who received concurrent treatment ${ }^{60}$. Although the study reports that treatment-related adverse events were generally manageable with immunosuppressants, these findings warrant further exploration into strategies which limit treatment-related adverse events.

\subsubsection{Checkpoint inhibitors combined with other immunotherapies}

Immune checkpoint blockade therapy may also increase the efficacy of vaccination and antibody-mediated therapies. Early vaccination with GM-CSF-expressing glioma cells and treatment with anti-CTLA4 antibodies resulted in increased survival of mice bearing GL261 tumors compared to either treatment alone ${ }^{61}$. In this model, combination therapy resulted in a greater antigen-specific immune response, as measured by IFN- $\gamma$ release by splenocytes upon 
stimulation with irradiated GL261 cells, when compared to monotherapy with CTLA4 blockade or vaccination alone. Other murine studies of immune checkpoint blockade combined with vaccination in different tumor models show similarly promising results ${ }^{62,63}$. A clinical study of nivolumab with a multipeptide vaccine in patients with ipilimumab-naïve or ipilimumabrefractory melanoma demonstrated safety and tolerability of treatment and revealed a response rate of $25 \%$ according to RECIST criteria ${ }^{64}$ The AVeRT trial (NCT02529072) is currently studying the safety and efficacy of nivolumab with and without DC vaccine therapy prior to surgical resection and following surgery. Other clinical trials are testing immune checkpoint blockade in combination with antibody therapy directed at alternative targets. A phase 1 study is currently testing nivolumab in combination with the anti-CSF1R antibody FPA008, which blocks recruitment of tumor-associated macrophages to the tumor microenvironment in patients with advanced cancers, including glioma (NCT02526017).

Checkpoint inhibition has also been shown to improve the efficacy of CAR T cell therapy. Chimeric antigen receptors (CARs) are synthetic molecules that re-direct $\mathrm{T}$ cells to specific antigens that are ideally not expressed on normal tissues ${ }^{65}$. However, the success of CAR T cell therapy has been limited by several factors including immunosuppressive tumor microenvironment, tumor heterogeneity, and limited $\mathrm{T}$ cell trafficking to tumor $\operatorname{sites}^{66,67}$. A preclinical study aimed to target immunosuppression mediated by upregulation of immunosuppressive checkpoint ligands in tumor microenvironment in order to enhance cytotoxic (efficacy of CAR T cells ${ }^{68}$. CAR T cells targeting costimulatory domains CD28 and CD4-1BB in a mouse model of pleural mesothelioma showed decreased $\mathrm{T}$ cell lytic function in the presence of upregulated PD-1. PD-1 antibody blockade in combination with CD28-targeted CAR T cell therapy rescued exhausted CD 28 CAR T cells ${ }^{68}$. Additionally, CD28 CAR T cells engineered to 
block PD-1 signaling exhibited enhanced tumor control and median survival when compared to the unmodified CD28 CAR T cell control in a mouse model of pleural mesothelioma ${ }^{68}$.

3.2 Other combination strategies targeting immunosuppression in the tumor microenvironment

Some approaches aim to limit tumor immunosuppression by targeting immunosuppressive myeloid cells, such as TAMs or MDSCs. Preclinical studies by Kosaka et al. investigated the effects of combination therapy with a CD40 agonist mAb, which has been shown to activate myeloid cells and induce myeloid cell polarization towards a tumoricidal phenotype, and the COX-2 inhibitor celecoxib, which inhibits immunosuppressive functions of MDSCs ${ }^{69}$. This study revealed that combination therapy with the $\mathrm{CD} 40 \mathrm{mAb}$ and celecoxib resulted in increased survival of mice bearing Quad-GL261 glioma cells when compared to monotherapy with either CD40 mAb or celecoxib alone. Furthermore, it was shown that only combination therapy resulted in increased production of IFN- $\gamma$ by CD4 $+\mathrm{T}$ cells and reduced proportions of $\mathrm{T}_{\text {regs, }}$, suggesting immunostimulatory effects beyond inhibition of immunosuppressive myeloid cells ${ }^{69}$. MDSC inhibition may also improve the efficacy of vaccination therapy. Patients with glioblastoma show increased serum concentrations of CD200, a glycoprotein significantly correlated with MDSC expansion through its interaction with $\mathrm{CD}_{200 \mathrm{R}^{70}}$. Combination of a CD200R antagonist with tumor lysate vaccination in GL261 glioma bearing mice resulted in reduced tumor growth and enhanced survival when compared to vaccination with tumor lysate alone $^{70}$.

Inhibition of immunosuppression in the tumor microenvironment can also enhance the antitumor effects of adoptive immunotherapies such as cytotoxic T lymphocyte (CTL) transfer. CTL transfer involves isolation of CTLs from the host patient, activation and expansion ex vivo, 
and re-introduction of CTLs into the patient in order to target tumor cells $\mathrm{s}^{46,71,72}$. The success of CTL therapy has been hampered by immunosuppressive factors in the tumor microenvironment such as TGF- $\beta$, which has been shown to mediate immunosuppression through upregulation of miR-23a, an inhibitor of CTL cytotoxicity ${ }^{73}$. Combination of miR-23a inhibition with CTL therapy resulted in increased CTL expression of cytotoxic mediators IFN- $\gamma$ and granzyme-b and slowed tumor progression in mouse melanoma and lung cancer models ${ }^{74}$. The resistance of miR23a inhibited CTLs to TGF- $\beta$-mediated immunosuppression opens the possibility for CTL therapy enhancement in glioma models.

\section{3 Gene therapy strategies}

\subsubsection{Combinations of multiple immunostimulatory gene therapies}

While oncolytic or cancer ablative gene therapy might not be readily classified as conventional immunotherapy, approaches which induce cell death are powerful stimulators of the immune system. Gene therapy-mediated cell death has been shown to cause the release of damage associated molecular patterns (DAMPs) which signal through Toll-like receptors (TLRs) to activate tumor-infiltrating DCs and promote a tumor-specific CD8+ T cell response ${ }^{75}$. Multiple strategies for inducing immunogenic cell death and thereby stimulating innate and adaptive immune responses may be combined in order to achieve better outcomes in GBM treatment. An ongoing phase I clinical trial (NCT01811992) combines direct tumor cell killing With herpes simplex virus 1-thymidine kinase (HSV1-TK), and immune stimulation with Flt3L, a cytokine that recruits DCs into the tumor microenvironment ${ }^{76}$. Both gene therapy arms are delivered by first generation adenoviral vectors (Ad.TK and Ad.Flt3L) that are injected into the tumor bed after surgical removal of the tumor mass. Each vector constitutively expresses the 
therapeutic transgene under the control of the human cytomegalovirus promoter. Treatment with Ad.TK is expected to kill proliferating tumor cells that remain after surgery, exposing tumor antigens and releasing proinflammatory DAMPs ${ }^{76}$. Simultaneously, treatment with Ad.Flt3L stimulates DC migration into the tumor, triggering a specific immune response against GBM cells that remain throughout the brain parenchyma.

Additional approaches combine HSV-TK delivery with other cytokines. Okada and colleagues evaluated a cytokine gene therapy against an intracranial glioma using 9L gliosarcoma cell lines stably transduced with murine IL4 cDNA (9L-IL4), HSV-TK (9L-Tk) or both (9L-IL4-Tk) ${ }^{77}$. These transduced tumor cell-mediated therapies were subcutaneously injected into rats bearing intracranial 9L tumors. Administration of 9L-IL4-Tk cells followed by treatment with ganciclovir completely protected the animals from a subsequent intracranial challenge and improved long-term surviyal. This preclinical approach was translated into two clinical protocols assessing safety, feasibility, and preliminary clinical activity ${ }^{78}$. In the first protocol, adult participants with recurrent GBM or anaplastic astrocytoma (AA) received gross total resection of tumors, followed by two injections of autologous fibroblasts retrovirally transfected with TFG-IL4-Neo-TK vector mixed with irradiated autologous glioma cells. Participants demonstrated encouraging immunological and clinical responses, including $\mathrm{CD} 4^{+}$ and $\mathrm{CD}^{+} \mathrm{T}$ cell infiltration as well as systemic $\mathrm{T}$-cell responses. Although there was no evidence of adverse effects, participants eventually succumbed to tumor recurrence. In the second protocol adult participants with newly diagnosed GBM or AA were subjected to surgical removal and radiation therapy, followed by two intradermal vaccinations with transfected fibroblasts mixed with autologous tumor lysate-loaded DCs. Treatment was well tolerated and participants showed high levels of IL-12 p70 produced by monocyte-derived DCs; however there 
was no detectable IFN- $\gamma$ post-vaccine response or prolonged progression free survival. Nevertheless, these findings warrant further investigation of combination therapies for GBM patients. Another ongoing Phase I clinical trial is testing the efficacy of oncolytic virotherapy with an oncolytic herpes simplex virus (oHSV) engineered to express IL-12 in patients with recurrent/progressive GBM, anaplastic astrocytoma, or gliosarcoma (NCT02062827) ${ }^{79}$. Oncolytic viruses are conditionally replicative viruses which selectively replicate within and kill tumor cells, exploiting differential protein expression profiles between tumor cells and normal cells. This cytokine-expressing oHSV combines direct tumor cytotoxicity with increased IL-12 expression, which has been shown to induce a Th-1 type immune response and enhance activity of NK cells and cytotoxic $\mathrm{T}$ cells, in order to target the tumor through multiple different pathways.

\subsubsection{Immunostimulatory gene therapy combined with other immunotherapies}

In a preclinical study, Mineharu et al showed a synergistic effect of immunostimulatory gene therapy combined with DC vaccination ${ }^{80}$. Rat glioma models were given an intratumoral injection of Ad-TK/Flt3L in combination with subcutaneous vaccination with DCs pulsed with tumor lysates. Combination treatment showed a remarkable increase of long-term survivors (90\%) compared to either treatment alone $(10 \%$ for DC vaccine and $50 \%$ for immunostimulatory gene therapy). It was speculated that intratumoral injection of immunostimulatory cytokines, such as Flt3L, prolonged the survival of subcutaneously administered DCs, increasing their antitumor potency.

Recent preclinical data from our group also provide evidence of enhanced therapeutic efficacy of TK/Flt3L gene therapy in combination with other immunotherapies. MDSC depletion 
with the anti-Gr-1 antibody following TK/Flt3L gene therapy resulted in $77 \%$ long term survival of mice bearing intracranial GBM compared to $50 \%$ survival with gene therapy alone ${ }^{81}$. Data from the same set of studies show that immunosuppressive checkpoint blockade combined with TK/Flt3L gene therapy yields similarly promising results: PDL1 blockade combined with TK/Flt3L gene therapy resulted in $80 \%$ long term survival of tumor-bearing mice compared to $50 \%$ survival from TK/Flt3L gene therapy alone, and CTLA-4 blockade combined with TK/Flt3L gene therapy resulted in 57\% long term survival of GBM bearing mice compared to $28 \%$ survival from TK/Flt3L gene therapy alone ${ }^{81}$. These studies indicate that activation of the immune system in combination with strategies which limit barriers to immune function upon activation may be promising strategies for clinical trials in the future.

\subsection{Combination strategies utilizing vaccination therapy}

\subsubsection{Vaccination combined with immune stimulatory adjuvants}

Immune stimulatory adjuvants such as GM-CSF and TLR agonists have been used to enhance vaccination-induced immune responses. In a phase I study of the IMA950 vaccine, a peptide vaccine containing 11 GBM-associated peptides, with GM-CSF, a cytokine which stimulates DC maturation, in newly diagnosed GBM patients, $90 \%$ of patients had a CD8+ T-cell immune response to at least one peptide, but the amplitude was low ${ }^{82}$. A new phase I/II study of the IMA950 vaccine (NCT01920191) includes intramuscular poly-ICLC, an agonist of TLR3 which is widely used as an immune adjuvant. Other TLR ligands, such as the TLR7 ligand imiquimod, augment GM-CSF-initiated antitumor immunity by activating plasmacytoid DCs and decreasing the population of $\mathrm{T}_{\text {regs }}{ }^{83}$. A phase I clinical trial (NOA-16) is testing an IDH1 mutant peptide vaccine in combination with imiquimod in patients with IDH1 R132H mutant grade III and IV tumors without 1p/19q co-deletion and with ATRX loss (NCT02454634). 
STING ligand, another adjuvant and stimulator of IFN production, has been shown to enhance the efficacy of peptide vaccination in glioma bearing mice ${ }^{84}$.

Inhibition of the mTOR pathway has been reported to augment effector and memory CD8+ $\mathrm{T}$ cell functions ${ }^{85}$. However, the use of mTOR inhibitors, such as rapamycin, as immune adjuvants in cancer immunotherapy has yielded conflicting results. Some animal studies show that mTOR inhibitors augment therapeutic efficacy of heat shock protein cancer vaccine and immuno-gene therapy for glioma ${ }^{86,87}$, while others report that mTOR inhibitors attenuate the therapeutic efficacy of peptide vaccination ${ }^{88}$. It is not yet known whether vaccine therapy is more efficacious when administered with rapamycin in treating solid tumors in humans. A clinical trial is testing the ability of mTOR inhibition with rapamycin to enhance DC vaccine (CDX-1401, DEC205/NY-ESO-1 fusion) induced anti-tumor immunity in patients with NY-ESO-1 expressing solid tumors including glioma (NCT01522820).

It has also been demonstrated that pre-conditioning the vaccine site with tetanus/diphtheria toxoid can significantly improve the efficacy of DC vaccines in both preclinical and clinical settings ${ }^{89}$. The randomized blinded clinical trial of a DC vaccine pulsed with cytomegalovirus phosphoprotein 65 RNA in combination with tetanus/diphtheria toxoid vaccination site preconditioning prolonged the survival of patients with GBM, half of them surviving longer than 40 months $^{89}$. An ongoing phase 1 study (RESIST, NCT02193347) testing the safety and immunogenicity of the IDH1 peptide vaccine (PEPIDH1M) in patients with recurrent grade II glioma positive for IDH1R132H includes the administration of IDH1 mutant peptide emulsified with the water in oil emulsion Montanide ISA-51 in combination with GM-CSF and tetanus toxoid preconditioning at the vaccination site. Combination of immune adjuvants Montanide 
ISA-51 and GM-CSF has been shown to induce cytotoxic immune reactions in both preclinical ${ }^{90}$ and clinical studies ${ }^{91-93}$.

\subsubsection{Vaccination combined with other immunotherapies}

One option for combination therapy that is under investigation in multiple solid tumors is the use of DC vaccination alongside adoptive $\mathrm{T}$ cell transfer. This concept derives from the principle that while DC vaccine monotherapy generally has acceptable toxicity, the immune response is often gradual and clinical efficacy frequently sub-optimal ${ }^{46,72,94}$. Similarly, efforts to perform isolated adoptive $\mathrm{T}$ cell transfer for solid tumors often result in a rapid decrease in the persistence of the infused T-cells ${ }^{94}$. Therefore early work combining these therapies has involved optimizing the time interval between $\mathrm{DC}$ vaccination and $\mathrm{T}$ cell transfer. In preclinical work performed in adenocarcinoma models, it was shown that vaccination with CEApeptide pulsed DCs within one day of adoptive transfer of CEA-specific T cells resulted in synergistic tumor killing ${ }^{94}$. This result was not seen when the DC vaccine was administered even three days after T cell transfer ${ }^{94}$. Similarly, the combination of tumor antigen loaded DCs with lymph node deriyed $\mathrm{T}$ cells has proven efficacious in pre-clinical models of mammary carcinoma and melanoma ${ }^{95,96}$. The feasibility and safety of this approach was demonstrated in a Phase I clinical trial of patients with metastatic melanoma ${ }^{97}$. While not yet tested specifically in human GBM patients, this approach is currently being studied in a clinical trial in pediatric patients with refractory medulloblastoma (NCT01326104). In this trial, DCs and autologous lymphocytes are loaded with total tumor RNA (TTRNA). The lymphocytes are then infused intravenously along with multiple doses of intradermal DC vaccination. 
Vaccination therapies for GBM have also been combined with anti-angiogenic monoclonal antibody therapy. Bevacizumab is a recombinant humanized monoclonal antibody targeting vascular endothelial growth factor A (VEGF-A), inhibiting new blood vessel formation. GBM is a highly vascular tumor and strongly expresses VEGF, prompting initial enthusiasm for its use as a therapeutic agent ${ }^{98,99}$. Unfortunately, bevacizumab therapy has not improved overall survival in GBM, despite some evidence of providing improved progression-free suryival ${ }^{99}$. Combining anti-angiogenic therapy with immune-based therapies may have synergistic impact; antiangiogenic therapy normalizes the tumor vasculature, promoting better infiltration of immune cells into the tumor mass and resulting in immune-mediated tumor death ${ }^{100,101}$. A current trial is investigating whether the combination of bevacizumab and Rindopepimut (a vaccine comprising the EGFRvIII-specific peptide sequence conjugated to keyhole limpet hemocyanin (KLH), a carrier protein) will prove efficacious in relapsed EGFRvIII-positive GBM (NCT01498328). Another trial in relapsed GBM is exploring the use of bevacizumab in combination with a vaccine derived from autologous tumor heat shock protein-peptide complexes which deliver tumor antigens to DCs (NCT01814813) ${ }^{102}$.

4. Chemotherapy and immunotherapy

Standard of care TMZ chemotherapy has been shown to cause profound lymphopenia and myelosuppression ${ }^{103}$, leading some to predict that TMZ-induced lymphodepletion reduces the antitumor efficacy of immunotherapies. Surprisingly, growing evidence suggests that chemotherapy may potentiate immunotherapy for glioma. Chemotherapy-induced lymphodepletion has been shown to increase cytokines IL-2, IL-7, IL-15, and BlyS, which facilitate clonal expansion of $\mathrm{B}$ and $\mathrm{T}$ cells $\mathrm{s}^{104}$. The immune reconstitution response also triggers increased number and activation of APCs and promotes strong cellular and humoral responses ${ }^{104}$. 
Thus immune reconstitution may synergize with immunotherapy, strengthening antitumor immune response. In a preclinical study, high dose myeloablative TMZ followed by adoptive $\mathrm{CD} 8+\mathrm{T}$ cell transfer and peptide vaccination in a mouse brain tumor model resulted in 70 fold expansion of antigen-specific CD8+ T cells over the control group which did not receive TMZ therapy $^{105}$. In a phase II multicenter trial the EGFRvIII peptide vaccine was administered with concomitant standard or dose intensified (DI) TMZ in patients with GBM. Although patients in the DI cohort had greater lymphopenia and an increased proportion of $\mathrm{T}_{\text {regs, }}$ the DI cohort showed a greater magnitude of humoral and delayed-type hypersensitivity responses when compared to the standard cohort ${ }^{106}$. Alternatively, design of chemotherapy-resistant immunotherapies may improve safety and efficacy of treatment regimens. $\gamma \delta$ Tcells, which are genetically modified to resist TMZ, demonstrated greater cytotoxicity against TMZ resistant cell lines in the presence of TMZ than unmodified $\gamma \delta$ Tcells, opening the possibility for combined administration of chemotherapy and cellular immunotherapy products ${ }^{107}$. Other approaches utilize chemotherapy-resistant hematopoietic stem cells to circumvent the toxicities of severe chemotherapy-induced lymphopenia caused by O6-benzylguanine and $\mathrm{TMZ}^{108,109}$. Such studies are only a small sample of the many promising strategies which leverage the immunostimulatory effects of myeloablative chemotherapy while minimizing off-target cellular toxicities.

\section{Caveats to combination immunotherapy}

Combinations of immunotherapies have the potential to enhance antitumor immune response and bypass tumor-mediated immunosuppression. Nevertheless, some combinations could result in reduced antitumor effect or adverse systemic effects. As mentioned earlier, combinations of immune checkpoint inhibitors could elicit systemic adverse events related to exacerbated and deleterious auto-immune responses ${ }^{60}$. Additionally, immunostimulatory therapy may blunt the 
efficacy of oncolytic virotherapy, which depends on direct tumor cytotoxicity. Although the mechanisms by which oncolytic viruses lead to tumor rejection remain elusive, antitumor immunity seems to play a key role in addition to direct cytotoxic effect ${ }^{110}$. Release of tumor antigens and DAMPs upon tumor cell lysis has been involved in the maturation of antigenpresenting cells, amplifying the antitumor effect of oncolytic therapy in non-infected tumor cells ${ }^{110}$. It is certainly a possibility that activation of an antiviral immune response could lead to the destruction of cells harboring the oncolytic viruses prior to tumoricidal and immunogenic effect. The oncolytic polio virus PVSRIPO virus is particularly effective in evading premature elimination by the host innate immune response; PVSRIPO demonstrates impressive cytotoxic effects through dysregulated mitogenic signaling networks which are relatively unaffected by innate antiviral responses ${ }^{11,112}$. As oncolytic virotherapies improve in cytotoxic efficacy and we begin to elucidate the role of the host immune response in oncolytic virotherapy it may be possible to design combinations which minimize opposing immune effects.

\section{Conclusion}

Growing evidence suggests that the modest success of single immunotherapeutic strategies in clinical trials for glioblastoma might be significantly improved by combining multiple approaches. Targeting of multiple antigens, immune-stimulatory/inhibitory pathways, and immune cell populations has the potential to effectively target heterogeneous populations of tumor cells and overcome the immunosuppressive mechanisms employed by GBM, inducing synergistic antitumor responses. Combination of immunotherapies with traditional cytotoxic therapies, i.e. radiation and TMZ, demonstrated remarkable success in preclinical studies; this provides a compelling rationale for testing these combined therapeutic strategies in GBM patients. 


\section{Expert opinion}

Glioma is one of the most challenging to treat human cancers, and patients have a dismal prognosis in spite of improved standard of care, which includes surgery, radiation and chemotherapy $^{2,3}$. Several compounding factors complicate the treatment of this primary brain cancer. Most notably, at the time of diagnosis the tumor is already very large (due to the lack of early symptoms), highly invasive, and is histologically and molecularly heterogeneous ${ }^{17}$. The blood brain barrier limits the penetration of chemotherapeutic agents into the tumor, thus rendering most anti-cancer agents are ineffective. In addition, antigen presenting cells are scarce in the brain parenchyma; thus, it is not possible to mount an effective anti-tumor immune response to tumors which are located in the central nervous system ${ }^{4-7}$. There are numerous experimental strategies that are currently being developed to treat GBM, one of the most exciting being immunotherapy, as described here. In spite of a concerted effort from many investigators throughout the world, single arm immunotherapeutic strategies, in which patients exhibit positive CD8 $\mathrm{T}$ cell responses, have not resulted in significant improvements in overall survival. These include DC vaccination strategies, single tumor specific antigen-based vaccines (i.e., EGFRvIII), tumor antigen specific CAR-T cells, oncolytic virotherapy, and single arm gene therapies, as reviewed earlier.

With many of the above mentioned approaches, pre-clinical efficacy data generated from animal models of GBM has not been able to predict efficacy in human patients. There are many reasons for this, but the most critical include: the use of flank tumor models that do not mimic the brain anatomical and physiological microenvironment, the use of homogeneous tumor cells over-expressing the single antigen of choice, treatment of very small tumors which are not representative of the clinical scenario, lack of testing in models of GBM recurrence, and using 
statistically significant, albeit biologically small effect-size differences in median survival as an indication that a particular therapeutic approach could be successful in Phase I clinical trials. The field is now beginning to address some of these issues, with the development of more stringent models that enable testing of experimental immunotherapies in large and heterogeneous brain tumor models, using models of GBM recurrence, and working towards treatments that start at the time the animal becomes symptomatic due to tumor burden. In addition, the consensus is that due to the highly heterogeneous nature and the rapid rate of mutation of GBM tumor cells, the use of a single therapeutic modality will not prove to be efficacious against this disease ${ }^{26,27}$. Thus, several groups, including our own, are moving towards combination therapies. To that effect, our group has pioneered a gene therapy-mediated combined conditional cytotoxic immune stimulatory strategy that is currently being tested in human patients with GBM. This combination immune-stimulatory gene therapy Phase I trial is entitled: "A non-randomized, open-label dosefinding trial of combined cytotoxic and immune-stimulatory strategy for the treatment of primary GBM, utilizing adenovirus vector type 5 (Ad-hCMV-TK) expressing herpes simplex virus thymidine kinase, and Ad vector type 5 (Ad-hCMV-Flt3L) expressing fms-like tyrosine kinase ligand" and is currently ongoing at our institution (IND number BB14574, clinicaltrials.gov number NCT01811992).

We hope that this review article will encourage testing of novel immunotherapeutic approaches which combine several targets, i.e., vaccination using multiple tumor epitopes, combining vaccination or gene therapy strategies with immune checkpoint blockade, or combining CAR-T cells with either vaccination and/or immune checkpoint inhibitors, amongst several other possibilities. The fact that concomitant radiotherapy and chemotherapy has shown to improve the antitumor efficacy of immunotherapeutic strategies encourages the incorporation 
of immunotherapeutic strategies to routine treatment modalities in GBM patients. In addition, the field should continue to move towards more stringent animal models that more closely recapitulate the clinical scenario and only take as a positive indication of efficacy large efficacy size-effects in improvements in median survival as opposed to marginal improvements of just a few days. As a field, we have advanced tremendously over the past decade; still, much work remains to be done to provide meaningful therapeutic outcomes to patients suffering from this devastating brain cancer.

\section{Acknowledgements:}

The authors are thankful for the academic leadership and support received from Dr. Karin Muraszko and the Department of Neurosurgery; to A. Collada and S. Napolitan for superb administrative support; to M. Dzaman for outstanding technical assistance; and Elijah Lowenstein for superb editorial comments.

\section{Funding:}

This work was supported by: National Institutes of Health/National Institute of Neurological Disorders \& Stroke (NIH/NINDS) Grants R37-NS094804, R01-NS094804, R01-NS074387, R01-NS057711, R21-NS091555, and R01-NS094804 to MG Castro.; NIH/NINDS Grants R01NS061107, R01-NS076991, R01-NS082311, and R21-NS084275 to PR Lowenstein.; Leah's Happy Hearts, University of Michigan Comprehensive Cancer Center, Chad Tough Foundation, and The Phase One Foundation to both MG Castro. and PR Lowenstein. It was also supported by: the Department of Chemical Engineering, University of Michigan (S01-OD020053); the Department of Neurosurgery, University of Michigan School of Medicine; the Michigan Institute for Clinical and Health Research (under NIH grant 2UL1-TR000433); University of Michigan 
Cancer Biology Training Grant, NIH/NCI (National Cancer Institute) grant T32-CA009676; the University of Michigan Training in Clinical and Basic Neuroscience through NIH/NINDS grant T32-NS007222; the University of Michigan Medical Scientist Training Program, and NIH/NIGMS (National Institute of General Medicine Sciences) grant T32-GM007863. Finally, the authors are given support by the National Council of Scientific and Technological Research (CONICET, Argentina, PIP 2011-00353 to M Candolfi and a Doctoral Fellowship to AS.Asad) and the National Agency for the Promotion of Science and Technology (ANPYCT, Argentina, PICT 2013-0310, PICT 2015-3309 to M Candolfi.).

\section{Declaration of interest:}

The authors have no relevant affiliations or financial involvement with any organization or entity with a financial interest in or financial conflict with the subject matter or materials discussed in the manuscript. This includes employment, consultancies, honoraria, stock ownership or options, expert testimony, grants or patents received or pending, or royalties. 


\section{Figure legends:}
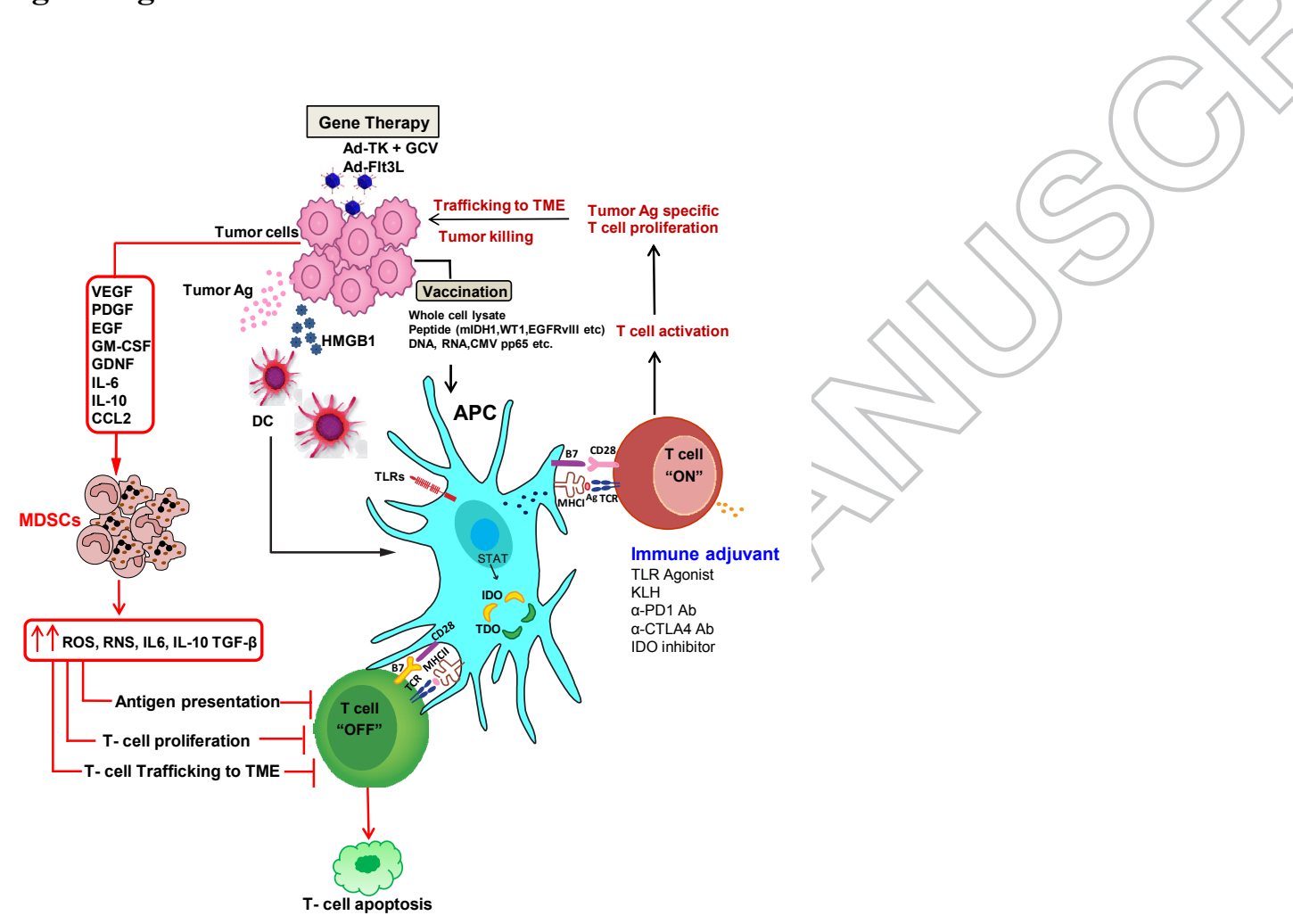

Figure 1. Adenoviral-mediated TK/FIt3L gene therapy and tumor-induced immune suppression.

Intra-tumoral injections of adenovirus expressing TK in combination with adenovirus expressing Flt3L induce tumor cell death and release of intracellular inflammatory molecules, such as 
HMGB1 and tumor antigen. Flt3L recruits DCs to the tumor site, where they phagocytize tumor cell remnants and migrate to the draining lymph node. Priming, activation and proliferation of tumor Ag-specific $\mathrm{T}$ cells in the lymph node result in a $\mathrm{T}$ cell-mediated cytotoxic anti-tumor immune response.

Glioma cells produce cytokines (VEGF, PDGF, LIF, GDNF, IL-6, IL-10, CCL2) which promote differentiation towards immunosuppressive phenotypes and increase the number of Myeloid Derived Suppressor Cells (MDSCs). MDSCs are recruited to the tumor microenyironment and circulate back to lymphoid organs to suppress anti-tumor immune responses. These MDSCs impair differentiation, maturation, activation, and proliferation of T cells, eventually leading to $\mathrm{T}$ cell death.

Abbreviations used: GCV, Ganciclovir; TK, thymidine kinase; Flt3L, fms-like tyrosine kinase 3 ligand; HMGB1, high-mobility group box-1; Ag, antigen, DC, dendritic cells; APC, antigen presenting cells; TLR, toll like receptor; CTLA-4, cytotoxic T-lymphocyte antigen 4; IL, interleukin; MDSC, myeloid-derived suppressor cell; MHC, major histocompatibility class; TCR, T cell receptor; TLR, toll-like receptor; CCL2, Chemokine ligand 2 (also known as monocyte chemotactic protein -1 MCP-1); EGF, epidermal growth factor; GDNF, glial derived neurotrophic factor; GM-CSF, granulocyte macrophage colony stimulating factor; iNOS, inducible nitric oxide synthase (also known as NOS2); PGE2, Prostaglandin E2; PDGF, plateletderived growth factor; TGF $\beta$, Transforming growth factor beta; VEGF, vascular endothelial growth factor; ROS, reactive oxygen; RNS, reactive nitrogen; TME, tumor microenvironment 


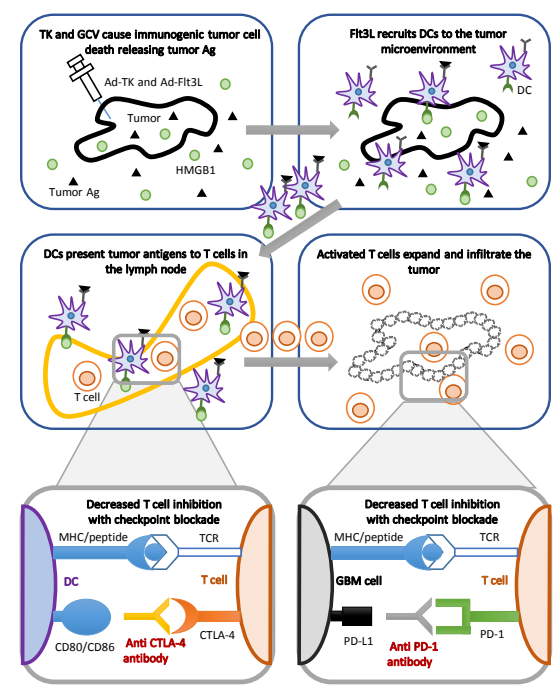

Figure 2. TK/FIt3L gene therapy combined with immunosuppressive checkpoint blockade. Administration of Ad-TK and ganciclovir (GCV) results in tumor cell death and release of the pro-inflammatory ligand HMGB1 and tumor antigen (Ag). Ad-Flt3L encodes the cytokine Flt3L which recruits dendritic cells (DCs) into the tumor microenvironment. DCs take up tumor antigens, migrate to the lymph node, and present tumor antigens to $\mathrm{T}$ cells in the lymph node. Administration of immunosuppressive checkpoint blockade therapy such as anti-CTLA4 antibodies ipilimumab or tremelimumab results in decreased $\mathrm{T}$ cell inhibition. Activated $\mathrm{T}$ cells migrate to the tumor microenvironment and kill tumor cells. Administration of immunosuppressive checkpoint blockade therapies such as anti-PD1/PDL1 antibodies nivolumab, pembrolizumab, or pidilizmab results in decreased GBM-mediated $\mathrm{T}$ cell inhibition and augments $\mathrm{T}$ cell cytotoxicity. 


\section{References}

1. Network TCGAR. Comprehensive, Integrative Genomic Analysis of Diffuse Lower-Grade Gliomas. New England Journal of Medicine 2015;372(26):2481-98.

2. Ostrom QT, Bauchet L, Davis FG, et al. The epidemiology of glioma in adults: a "state of the science" review. Neuro-Oncology 2014;16(7):896-913.

3. Stupp R, Mason WP, van den Bent MJ, et al. Radiotherapy plus Concomitant and Adjuvant Temozolomide for Glioblastoma. New England Journal of Medicine 2005;352(10):987-96.

4. Lowenstein PR, Kroeger K, Castro MG. Immunology of Neurological Gene Therapy: How T Cells Modulate Viral Vector-Mediated Therapeutic Transgene Expression Through Immunological Synapses. Neurotherapeutics : the journal of the American Society for Experimental NeuroTherapeutics 2007;4(4):715-24.

5. Barcia C, Jimenez-Dalmaroni M, Kroeger KM, et al. One-year Expression From High-capacity Adenoviral Vectors in the Brains of Animals With Pre-existing Anti-adenoviral Immunity: Clinical Implications. Mol Ther 2007;15(12):2154-63.

6. Barcia C, Thomas CE, Curtin JF, et al. In vivo mature immunological synapses forming SMACs mediate clearance of virally infected astrocytes from the brain. The Journal of Experimental Medicine 2006;203(9):2095-107.

7. Lowenstein PR, Castro MG. Recent advances in the pharmacology of neurological gene therapy. Current opinion in pharmacology 2004;4(1):91-97.

8. Galea I, Bernardes-Silva M, Forse PA, et al. An antigen-specific pathway for CD8 T cells across the blood-brain barrier. The Journal of Experimental Medicine 2007;204(9):2023-30.

9. Klein RS, Garber C, Howard N. Infectious immunity in the central nervous system and brain function. Nat Immunol 2017;18(2):132-41.

10. Savarin C, Bergmann Cornelia C, Hinton David R, et al. MMP-independent role of TIMP-1 at the blood brain barrier during viral encephalomyelitis. ASN NEURO 2013;5(5):e00127.

11. Davies DC. Blood-brain barrier breakdown in septic encephalopathy and brain tumours. Journal of Anatomy 2002;200(6):639-46.

12. D'Agostino PM, Gottfried-Blackmore A, Anandasabapathy N, et al. Brain dendritic cells: biology and pathology. Acta Neuropathologica 2012;124(5):599-614.

13. Louveau A, Harris TH, Kipnis J. Revisiting the Mechanisms of CNS Immune Privilege. Trends in Immunology 2015;36(10):569-77.

14. Perng P, Lim M. Immunosuppressive Mechanisms of Malignant Gliomas: Parallels at Non-CNS Sites. Frontiers in Oncology 2015;5:153.

15. Nduom EK, Weller M, Heimberger AB. Immunosuppressive mechanisms in glioblastoma. NeuroOncology 2015;17(suppl 7):vii9-vii14.

16. Appin CL, Brat DJ. Biomarker-driven diagnosis of diffuse gliomas. Molecular Aspects of Medicine 2015; 45:87-96.

17. Louis DN, Perry A, Reifenberger G, et al. The 2016 World Health Organization Classification of Tumors of the Central Nervous System: a summary. Acta Neuropathologica 2016;131(6):803-20.

18. * *ai AA. Evidence for sequenced molecular evolution of IDH1 mutant glioblastoma from a distinct cell of origin. Journal of clinical oncology 2011;29(34):4482-90.

Provides evidence that IDH1 mutant GBM arises from a unique cell type of origin and represents a different disease entity than IDH1 wild type GBM.

19. Ohgaki HH. The definition of primary and secondary glioblastoma. Clinical cancer research 2013;19(4):764-72. 
20. Paugh B. Integrated molecular genetic profiling of pediatric high-grade gliomas reveals key differences with the adult disease. Journal of clinical oncology 2010;28(18):3061-68.

21. Sturm D, Bender S, Jones DTW, et al. Paediatric and adult glioblastoma: multiform (epi)genomic culprits emerge. Nat Rev Cancer 2014;14(2):92-107.

22. Fontebasso AM, Liu X-Y, Sturm D, et al. Chromatin Remodeling Defects in Pediatric and Young Adult Glioblastoma: A Tale of a Variant Histone 3 Tail. Brain Pathology 2013;23(2):210-16.

23. Siegal T. Clinical Relevance of Prognostic and Predictive Molecular Markers in Gliomas. In: Schramm J, ed. Advances and Technical Standards in Neurosurgery: Volume 43. Cham: Springer International Publishing 2016:91-108.

24. *Koschmann CC. ATRX loss promotes tumor growth and impairs nonhomologous end joining DNA repair in glioma. Science translational medicine 2016;8(328):328ra28-28ra28.

Shows that ATRX deficiency in a mouse model of GBM results in genetic instability and increased responsiveness to double-stranded DNA-damaging agents.

25. Verhaak RGW, Hoadley KA, Purdom E, et al. Integrated Genomic Analysis Identifies Clinically Relevant Subtypes of Glioblastoma Characterized by Abnormalities in PDGFRA, IDH1, EGFR, and NF1. Cancer Cell 2010;17(1):98-110.

26. Patel AP. Single-cell RNA-seq highlights intratumoral heterogeneity in primary glioblastoma. Science (New York, NY) 2014;344(6190):1396-401.

27. Sottoriva A, Spiteri I, Piccirillo SGM, et al. Intratumor heterogeneity in human glioblastoma reflects cancer evolutionary dynamics. Proceedings of the National Academy of Sciences of the United States of America 2013;110(10):4009-14.

28. Pellegatta S, Cuppini L, Finocchiaro G. Brain cancer immunoediting: novel examples provided by immunotherapy of malignant gliomas. Expert Review of Anticancer Therapy 2011;11:1759+.

29. *Sampson JH, Heimberger AB, Archer GE, et al. Immunologic Escape After Prolonged Progression-Free Survival With Epidermal Growth Factor Receptor Variant III Peptide Vaccination in Patients With Newly Diagnosed Glioblastoma. Journal of clinical oncology 2010;28(31):4722-29. Demonstrates that administration of an EGFRvIII-specific peptide vaccine results in an antigen specific immune response and significant reduction in EGFRvIll expression upon tumor recurrence.

30. Ohm JE, Carbone DP. VEGF as a mediator of tumor-associated immunodeficiency. Immunologic Research 2001;23(2):263-72.

31. Qiu B, Zhang D, Wang C, et al. $1 \mathrm{~L}-10$ and TGF- $\beta 2$ are overexpressed in tumor spheres cultured from human gliomas. Molecular Biology Reports 2011;38(5):3585-91.

32. Wainwright DADA. IDO expression in brain tumors increases the recruitment of regulatory $T$ cells and negativelyimpacts survival. Clinical cancer research 2012;18(22):6110-21.

33. Fecci PE. Increased regulatory T-cell fraction amidst a diminished CD4 compartment explains cellular immune defects in patients with malignant glioma. Cancer research (Chicago, III) 2006;66(6):3294-302.

34. Wu A, WeiJ, Kong L-Y, et al. Glioma cancer stem cells induce immunosuppressive macrophages/microglia. Neuro-Oncology 2010;12(11):1113-25.

35. Gabrilovich DI, Nagaraj S. Myeloid-derived suppressor cells as regulators of the immune system. Nat Rev Immunol 2009;9(3):162-74.

36. Mirghorbani MM. Myeloid-derived suppressor cells in glioma. Expert review of neurotherapeutics 2013;13(12):1395-406.

37. Vladimir V. Didenko, Hop N. Ngo, Candace Minchew, et al. Apoptosis of T lymphocytes invading glioblastomas multiforme: a possible tumor defense mechanism. Journal of Neurosurgery 2002;96(3):580-84.

38. *Berghoff AS, Kiesel B, Widhalm G, et al. Programmed death ligand 1 expression and tumorinfiltrating lymphocytes in glioblastoma. Neuro-Oncology 2015;17(8):1064-75. 
Reports that PD-L1 expression and sparse to moderate density of TILs are found in a majority of GBM samples.

39. *Wintterle S, Schreiner B, Mitsdoerffer M, et al. Expression of the B7-Related Molecule B7-H1 by Glioma Cells. A Potential Mechanism of Immune Paralysis 2003;63(21):7462-67.

Shows that B7-H1, strongly expressed on glioma cells but not found in normal brain tissues, is a strong inhibitor of CD4+ and CD8+ T cells.

40. Crespo J, Sun $\mathrm{H}$, Welling TH, et al. T cell anergy, exhaustion, senescence, and stemness in the tumor microenvironment. Current Opinion in Immunology 2013;25(2):214-21.

41. Brunet J-F, Denizot F, Luciani M-F, et al. A new member of the immunoglobulin superfamily-CTLA-4. Nature 1987;328(6127):267-70.

42. Takahashi T, Tagami T, Yamazaki S, et al. Immunologic Self-Tolerance Maintained by Cd25+Cd4+ Regulatory T Cells Constitutively Expressing Cytotoxic T Lymphocyte-Associated Antigen 4. The Journal of Experimental Medicine 2000;192(2):303.

43. *Egen JG, Kuhns MS, Allison JP. CTLA-4: new insights into its biological function and use in tumor immunotherapy. Nat Immunol 2002;3(7):611-18.

Summarizes the role of CTLA4 in the regulation of T cell response and presents the CTLA4/TCR axis as a target for cancer immunotherapy.

44. Schwartz RHR. Costimulation of T lymphocytes: the role of CD28, CTLA-4, and B7/BB1 in interleukin-2 production and immunotherapy. Cell (Cambridge) 1992;71(7):1065-68.

45. ** Linsley PSP. Human B7-1 (CD80) and B7-2 (CD86) bind with similar avidities but distinct kinetics to CD28 and CTLA-4 receptors. Immunity (Cambridge, Mass) 1994;1(9):793-801.

Reports that CD80 and CD86 show similar overall T cell receptor binding and costimulatory properties, but CD80 binds CTLA4 with greater strength than CD86 and dissociates from CTLA4 more slowly than CD86.

46. Calinescu AA, Kamran N, Baker G, et al. Overview of current immunotherapeutic strategies for glioma. Immunotherapy 2015;7(10):1073-104.

47. Reardon DA, Freeman G, Wu C, et al. Immunotherapy advances for glioblastoma. NeuroOncology 2014;16(11):1441-58.

48. Binder DC. Immunotherapy for cancer in the central nervous system: Current and future directions. Oncoimmunology 2016;5(2):e1082027.

49. Mitchell DA, Fecci PE, Sampson JH. Immunotherapy of malignant brain tumors. Immunological Reviews 2008;222(1):70-100.

50. Kamran N, Calinescu A, Candolfi M, et al. Recent advances and future of immunotherapy for glioblastoma. Expert Opinion on Biological Therapy 2016;16(10):1245-64.

51. Hatanpaa KJ, Burma S, Zhao D, et al. Epidermal Growth Factor Receptor in Glioma: Signal Transduction, Neuropathology, Imaging, and Radioresistance. Neoplasia (New York, NY) 2010;12(9):67584.

52. Schuster J, Lai RK, Recht LD, et al. A phase II, multicenter trial of rindopepimut (CDX-110) in newly diagnosed glioblastoma: the ACT III study. Neuro-Oncology 2015;17(6):854-61.

53. CelldexTherapeutics. Data Safety and Monitoring Board Recommends Celldex's Phase 3 Study of RINTEGA ${ }^{\circledast}$ (rindopepimut) in Newly Diagnosed Glioblastoma be Discontinued as it is Unlikely to Meet Primary Overall Survival Endpoint in Patients with Minimal Residual Disease. Press Release 2016.

54. Mineharu Y, Castro MG, Lowenstein PR, et al. Dendritic cell-based immunotherapy for glioma: multiple regimens and implications in clinical trials. Neurologia medico-chirurgica 2013;53(11):741-54. 55. Prins $\mathrm{RM}$, Soto $\mathrm{H}$, Konkankit $\mathrm{V}$, et al. Gene expression profile correlates with $\mathrm{T}$ cell infiltration and relative survival in glioblastoma patients vaccinated with dendritic cell immunotherapy. Clinical cancer research : an official journal of the American Association for Cancer Research 2011;17(6):160315. 
56. * Okada $\mathrm{H}$, Kalinski $\mathrm{P}$, Ueda $\mathrm{R}$, et al. Induction of $\mathrm{CD} 8+\mathrm{T}$-cell responses against novel gliomaassociated antigen peptides and clinical activity by vaccinations with \{alpha\}-type 1 polarized dendritic cells and polyinosinic-polycytidylic acid stabilized by lysine and carboxymethylcellulose in patients with recurrent malignant glioma. Journal of clinical oncology : official journal of the American Society of Clinical Oncology 2011;29(3):330-6.

Demonstrates the ability of a DC vaccine loaded with glioma-associated antigen epitopes to induce antigen-specific immune responses, and improve 12 month progression free status in patients with malignant glioma.

57. Phuphanich S, Wheeler CJ, Rudnick JD, et al. Phase I trial of a multi-epitope-pulsed dendritic cell vaccine for patients with newly diagnosed glioblastoma. Cancer immunology, immunotherapy: CII 2013;62(1):125-35.

58. Hdeib A, Sloan AE. Dendritic cell immunotherapy for solid tumors: evaluation of the DCVax (R) platform in the treatment of glioblastoma multiforme. CNS oncology 2015;4(2):63-9.

59. Wainwright D. Durable therapeutic efficacy utilizing combinatorial blockade against IDO, CTLA-4, and PD-L1 in mice with brain tumors. Clinical cancer research 2014;20(20):5290-301.

60. Wolchok JD, Kluger H, Callahan MK, et al. Nivolumab plus Ipilimumab in Advanced Melanoma. New England Journal of Medicine 2013;369(2):122-33.

61. Agarwalla P, Barnard Z, Fecci P, et al. Sequential Immunotherapy by Vaccination with GM-CSF Expressing Glioma Cells and CTLA-4 Blockade Effectively Treats Established Murine Intracranial Tumors. Journal of Immunotherapy (Hagerstown, Md : 1997) 2012;35(5):385-89.

62. *Duraiswamy JJ. Dual blockade of PD-1 and CTLA-4 combined with tumor vaccine effectively restores T-cell rejection function in tumors. Cancer research (Chicago, III) 2013;73(12):3591-603. Demonstrates that blockade of both PD-1 and CTLA-4 resulted in proliferation of antigen specific CD8+ T cells, reversing CD8+ T cell dysfunction.

63. Williams ELEL. Immunomodulatory monoclonal antibodies combined with peptide vaccination provide potent immunotherapy in an aggressive murine neuroblastoma model. Clinical cancer research 2013;19(13):3545-55.

64. Weber JSJS. Safety, efficacy, and biomarkers of nivolumab with vaccine in ipilimumab-refractory or -naive melanoma. Journal of clinical oncology 2013;31(34):4311-18.

65. Maus MV. Designing CAR T cells for glioblastoma. Oncoimmunology 2015;4(12):e1048956.

66. Sengupta S, Mao G, Gokaslan ZS, et al. Chimeric antigen receptors for treatment of glioblastoma: a practical review of challenges and ways to overcome them. Cancer Gene Ther 2016. 67. Kakarla S, Gottschalk S. CAR T cells for solid tumors: armed and ready to go? Cancer journal (Sudbury, Mass) 2014;20(2):151-55.

68. Cherkassky L, Morello A, Villena-Vargas J, et al. Human CAR T cells with cell-intrinsic PD-1 checkpoint blockade resist tumor-mediated inhibition. The Journal of Clinical Investigation 2016;126(8):3130-44.

69. Kosaka A, Ohkuri T, Okada H. Combination of an agonistic anti-CD40 monoclonal antibody and the COX-2 inhibitor celecoxib induces anti-glioma effects by promotion of type-1 immunity in myeloid cells and T-cells. Cancer Immunology, Immunotherapy 2014;63(8):847-57.

70. Moertel CL, Xia J, LaRue R, et al. CD200 in CNS tumor-induced immunosuppression: the role for CD200 pathway blockade in targeted immunotherapy. Journal for ImmunoTherapy of Cancer 2014;2(1):46.

71. Restifo NP, Dudley ME, Rosenberg SA. Adoptive immunotherapy for cancer: harnessing the T cell response. Nat Rev Immunol 2012;12(4):269-81.

72. Madany M, Thomas TM, Edwards L, et al. Immunobiology and immunotherapeutic targeting of glioma stem cells. Advances in experimental medicine and biology 2015;853:139-66. 
73. Lin R, Sampson JH, Li Q-J, et al. miR-23a blockade enhances adoptive T cell transfer therapy by preserving immune-competence in the tumor microenvironment. Oncoimmunology 2015;4(3):e990803. 74. Lin R, Chen L, Chen G, et al. Targeting miR-23a in CD8+ cytotoxic T lymphocytes prevents tumordependent immunosuppression. Journal of Clinical Investigation 2014;124:5352+.

75. ${ }^{* *}$ Curtin JF, Liu N, Candolfi M, et al. HMGB1 mediates endogenous TLR2 activation and brain tumor regression. PLoS Med 2009;6(1):e10.

Describes a novel mechanism of anti-GBM immune stimulation through HMGB1 relase from dying tumor cells, resulting in TLR2 signaling.

76. Candolfi M, Kroeger KM, Muhammad AK, et al. Gene therapy for brain cancer: combination therapies provide enhanced efficacy and safety. Curr Gene Ther 2009;9(5):409-21.

77. Okada H, Giezeman-Smits KM, Tahara H, et al. Effective cytokine gene therapy against an intracranial glioma using a retrovirally transduced IL-4 plus HSVtk tumor vaccine. Gene Ther 1999;6(2):219-26.

78. Okada $\mathrm{H}$, Lieberman FS, Walter KA, et al. Autologous glioma cell vaccine admixed with interleukin-4 gene transfected fibroblasts in the treatment of patients with malignant gliomas. J Transl Med 2007;5:67.

79. Patel DM, Foreman PM, Nabors LB, et al. Design of a Phase IClinical Trial to Evaluate M032, a Genetically Engineered HSV-1 Expressing IL-12, in Patients with Recurrent/Progressive Glioblastoma Multiforme, Anaplastic Astrocytoma, or Gliosarcoma. Human Gene Therapy Clinical Development 2016;27(2):69-78.

80. $* *$ Mineharu $\mathrm{Y}, \mathrm{King} \mathrm{GD}$, Muhammad AK, et al. Engineering the brain tumor microenvironment enhances the efficacy of dendritic cell vaccination: implications for clinical trial design. Clinical cancer research : an official journal of the American Association for Cancer Research 2011;17(14):4705-18. Reveals that immunostimulatory gene therapy with $A d-F I t 3 L / A d-T K$ potentiates $D C$ vaccination, resulting in a significant increase in survival when compared to each therapy alone.

81. Kamran N, Kadiyala P, Saxena M, et al. Immunosuppressive Myeloid Cells' Blockade in the Glioma Microenvironment Enhances the Efficacy of/mmune-Stimulatory Gene Therapy. Molecular Therapy 2016; http://dx.doi.org/10.1016/i.ymthe.2016.10.003.

82. Rampling R, Peoples S, Mulholland PJ, et al. A Cancer Research UK First Time in Human Phase I Trial of IMA950 (Novel Multipeptide Therapeutic Vaccine) in Patients with Newly Diagnosed Glioblastoma. Clinical cancer research 2016;22(19):4776.

83. Narusawa $M$, Inoue $H$, Sakamoto $C$, et al. TLR7 ligand augments GM-CSF-initiated antitumor immunity through activation of plasmacytoid dendritic cells. Cancer immunology research 2014;2(6):568-80.

84. Ohkuri T, Ghosh A, Kosaka A, et al. Protective role of STING against gliomagenesis: Rational use of STING agonist in anti-glioma immunotherapy. Oncoimmunology 2015;4(4):e999523.

85. Araki K, Turner AP, Shaffer VO, et al. mTOR regulates memory CD8 T-cell differentiation. Nature 2009;460(7251):108-12.

86. Wang Y, Wang XY, Subjeck JR, et al. Temsirolimus, an mTOR inhibitor, enhances anti-tumour effects of heat shock protein cancer vaccines. British journal of cancer 2011;104(4):643-52.

87. Mineharu Y, Kamran N, Lowenstein PR, et al. Blockade of mTOR signaling via rapamycin combined with immunotherapy augments antiglioma cytotoxic and memory T-cell functions. Molecular cancer therapeutics 2014;13(12):3024-36.

88. Chaoul N, Fayolle C, Desrues B, et al. Rapamycin Impairs Antitumor CD8+ T-cell Responses and Vaccine-Induced Tumor Eradication. Cancer research 2015;75(16):3279-91.

89. Mitchell DA, Batich KA, Gunn MD, et al. Tetanus toxoid and CCL3 improve dendritic cell vaccines in mice and glioblastoma patients. Nature 2015;519(7543):366-9. 
90. Pellegatta $\mathrm{S}$, Valletta L, Corbetta $\mathrm{C}$, et al. Effective immuno-targeting of the IDH1 mutation $\mathrm{R} 132 \mathrm{H}$ in a murine model of intracranial glioma. Acta neuropathologica communications 2015;3:4.

91. Karbach J, Gnjatic S, Bender A, et al. Tumor-reactive CD8+ T-cell responses after vaccination with NY-ESO-1 peptide, CpG 7909 and Montanide ISA-51: association with survival. International journal of cancer 2010;126(4):909-18.

92. Kostic $A$, Mihailovic $D$, Veselinovic $S$, et al. Tumor size and karyometric variables in brain astrocytoma. Journal of BUON : official journal of the Balkan Union of Oncology 2009;14(3):473-7. 93. Markovic SN, Suman VJ, Ingle JN, et al. Peptide vaccination of patients with metastatic melanoma: improved clinical outcome in patients demonstrating effective immunization. American journal of clinical oncology 2006;29(4):352-60.

94. Park MY, Kim CH, Sohn HJ, et al. The optimal interval for dendritic cell vaccination following adoptive $T$ cell transfer is important for boosting potent anti-tumor immunity. Vaccine 2007;25(42):7322-30.

95. Tamai $\mathrm{H}$, Watanabe $\mathrm{S}$, Zheng $\mathrm{R}$, et al. Effective treatment of spontaneous metastases derived from a poorly immunogenic murine mammary carcinoma by combined dendritic-tumor hybrid vaccination and adoptive transfer of sensitized T cells. Clinical immunology 2008;127(1):66-77.

96. Song S, Zhang K, You H, et al. Significant anti-tumour activity of adoptively transferred $\mathrm{T}$ cells elicited by intratumoral dendritic cell vaccine injection through enhancing the ratio of CD8(+) T cell/regulatory T cells in tumour. Clinical and experimental immunology 2010;162(1):75-83.

97. Poschke I, Lovgren T, Adamson L, et al. A phase I clinical trial combining dendritic cell vaccination with adoptive T cell transfer in patients with stage IV melanoma. Cancer immunology, immunotherapy : CII 2014;63(10):1061-71.

98. Baker GJ, Yadav VN, Motsch S, et al. Mechanisms of glioma formation: iterative perivascular glioma growth and invasion leads to tumor progression, VEGF-independent vascularization, and resistance to antiangiogenic therapy. Neoplasia 2014;16(7):543-61.

99. Khasraw M, Ameratunga MS, Grant R, et al. Antiangiogenic therapy for high-grade glioma. The Cochrane database of systematic reviews 2014(9):CD008218.

100. Zhang B, Bowerman NA, Salama JK, et al. Induced sensitization of tumor stroma leads to eradication of established cancer by T cells. J Exp Med 2007;204(1):49-55.

101. Li B, Lalani AS, Harding TC, et al. Vascular endothelial growth factor blockade reduces intratumoral regulatory $\mathrm{T}$ cells and enhances the efficacy of a GM-CSF-secreting cancer immunotherapy. Clinical cancer research: an official journal of the American Association for Cancer Research 2006;12(22):6808-16.

102. Bloch $O$, Crane CA, Fuks $Y$, et al. Heat-shock protein peptide complex-96 vaccination for recurrent glioblastoma. a phase II, single-arm trial. Neuro Oncol 2014;16(2):274-9.

103. Sengupta S, Marrinan J, Frishman C, et al. Impact of Temozolomide on Immune Response during Malignant Glioma Chemotherapy. Clinical and Developmental Immunology 2012;2012:831090.

104. Sanchez-Perez L, Suryadevara CM, Choi BD, et al. Leveraging chemotherapy-induced lymphopenia to potentiate cancer immunotherapy. Oncoimmunology 2014;3(7):e944054.

105. Sanchez-Perez LA, Choi BD, Archer GE, et al. Myeloablative Temozolomide Enhances CD8(+) TCell Responses to Vaccine and Is Required for Efficacy against Brain Tumors in Mice. PLoS ONE 2013;8(3):e59082.

106. Sampson JH, Aldape KD, Archer GE, et al. Greater chemotherapy-induced lymphopenia enhances tumor-specific immune responses that eliminate EGFRvIII-expressing tumor cells in patients with glioblastoma. Neuro-Oncology 2011;13(3):324-33.

107. Lamb LS, Bowersock J, Dasgupta A, et al. Engineered Drug Resistant $\gamma \delta$ T Cells Kill Glioblastoma Cell Lines during a Chemotherapy Challenge: A Strategy for Combining Chemo- and Immunotherapy. PLOS ONE 2013;8(1):e51805. 
108. Adair JE, Beard BC, Trobridge GD, et al. Extended Survival of Glioblastoma Patients After Chemoprotective HSC Gene Therapy. Science translational medicine 2012;4(133):133ra57-33ra57.

109. Adair JE, Johnston SK, Mrugala MM, et al. Gene therapy enhances chemotherapy tolerance and efficacy in glioblastoma patients. The Journal of Clinical Investigation 2014;124(9):4082-92.

110. Kaufman HL, Kohlhapp FJ, Zloza A. Oncolytic viruses: a new class of immunotherapy drugs. Nat Rev Drug Discov 2015;14(9):642-62.

111. Brown MC, Gromeier M. Oncolytic Immunotherapy Through Tumor-specific Translation and Cytotoxicity of Poliovirus. Discov Med 2015;19(106):359-65.

112. Brown MC, Dobrikova EY, Dobrikov MI, et al. Oncolytic polio virotherapy of cancer. Cancer 2014;120(21):3277-86. 